
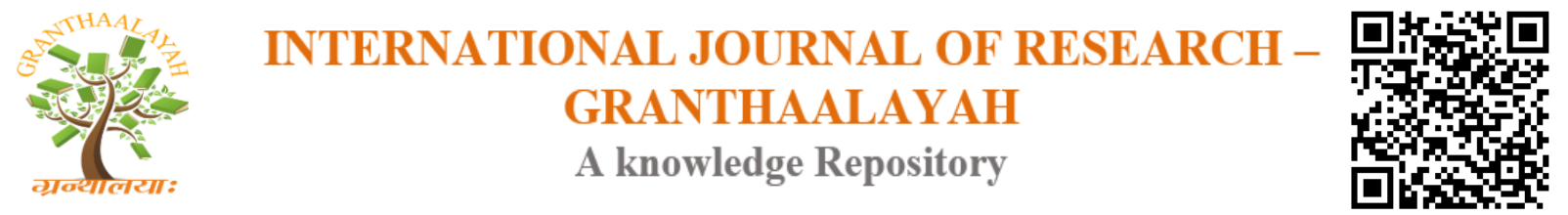

Science

\title{
GENOTYPE-SYMPTOMATOLOGY CORRELATION STUDIES IN PATIENTS WITH ANXIETY
}

\author{
Chen Shengdong ${ }^{1}$, Mingjun $\mathrm{He}^{2}$, Xiaoli $\mathrm{Zhu}^{2}$, Wei Niu ${ }^{3}$, Lingming Kong ${ }^{2}$, Gaofeng Chen ${ }^{2}$, \\ Liyi Zhang ${ }^{* 2}$ \\ ${ }^{* 1}$ Department of Neurology, No.102 Hospital of Chinese People's Liberation Army, Changzhou,
} People's Republic of China

${ }^{2}$ Prevention and Treatment Center for Psychological Diseases, No.102 Hospital of Chinese People's Liberation Army, Changzhou, People's Republic of China

${ }^{3}$ Department of Rehabilitation, No.102 Hospital of Chinese People's Liberation Army, Changzhou, People's Republic of China

\begin{abstract}
Projects: To explore the relationship of lncRNAs with altered expression in peripheral blood with symptomatogy in anxiety patients.

Methods: Gene microarray was carried on to screen the lncRNAs with altered expressions between anxiety patients (GAD) and healthy people (NC), and qPCR was performed to validate these screened lncRNAs. GAD was assessed by HAMA to analyze differentlyexpressed lncRNAs and its relationship with symptomatology.

Results: 1 . The expression levels of PR1-PR10 were positively relayed to psychic anxiety and the total score of HAMA $(\mathrm{r}=0.187 \sim 0.253, \quad P<0.01$ or $P<0.05)$, the expression level of PR7 was positively related to somatic anxiety $(\mathrm{r}=0.171, P<0.05) ; 2$. ROC curve combined analysis showed that the AUC value of ten lncRNAs arrived at 0.808 , at chic $\mathrm{YI}=\mathrm{YI} \mathrm{I}_{\max }$, sensitivity and specificity was $66.3 \%, 90.5 \%$ respectively; 3. High expression group of PR7 (NONHSAG049179) was significantly higher than that in low expression group accounting for psychic anxiety, aromatic anxiety and total score of HAMA.

Conclusion: IncRNA with altered expression may be involved in MDD, and NONHSAG049179 is closely associated with psychic anxiety and somatic anxiety.
\end{abstract}

Keywords: Anxiety; lncRNA; HAMA; Biomarker.

Cite This Article: Chen Shengdong, Mingjun He, Xiaoli Zhu, Wei Niu, Lingming Kong, Gaofeng Chen, and Liyi Zhang. (2017). "GENOTYPESYMPTOMATOLOGY CORRELATION STUDIES IN PATIENTS WITH ANXIETY." International Journal of Research-Granthaalayah, 5(12), 1-9. 


\section{Introduction}

Anxiety disorder (GAD) is a common alcoholism mental disorder, is characterized by nervous, fidget and some autonomic symptoms such as palpitations, handshakes, sweating, frequent urination and so on ${ }^{[1]}$. High morbidity $(0.8-2.7 \%)$, accompany with other mental disorder symptomatology bring pain to patients and damage their social function ${ }^{[2-3]}$.

No clear evidence has revealed the pathogenesis of GAD, but in a lot of researches, genetic factors, personality traits, cognitive processes, adverse life events and physical disease and so on were all indicated to be associated with GAD [4]. Therapies based on genetic and environmental factors were widely accepted and from the fields of neuroimaging, genomics, proteomics and immunomics, the technology of anxiety disorder etiology were explored [5], and it is confirmed that hereditary factor plays the leading role on the pathogenesis of anxiety disorder [6].

In 2002, long non-coding RNA (lncRNA) was discovered for first time, a molecular with a length of over 200nt, widely existing in animals, plants, yeasts, prokaryotes and viruses [8-10]. lncRNA can regulate genetic transcription through combining with promoters of genes which will interact with microRNA so as to motivate its biological function[13]. A numerous study recently indicated lncRNA's function in genetics due to its conservative secondary structures and shear forms. Because of the inconsistent between sequence and function of lncRNA, it is very hard to confirm their function. But fortunately, a large number of studies have indicated the association of IncRNA with schizophrenia, depression, alzheimer's disease, autism, so it is reasonable to speculate the regulation of lncRNA in anxiety.

Though its molecular and cellular pathophysiology has not been completely elucidated, it has been suggested that nearly $70 \%-85 \%$ of the risk for psychonosema is contributed to genetic factors, so the genetic and hereditary strategies are routine ways to evaluated these potential mechanism of anxiety. In this study, through long non-coding RNA (lncRNA) expression profiling and reverse transcription-polymerase chain reaction, 426 down-regulated and 120 upregulated.

\section{Materials and methods}

\subsection{Subjects}

69 GAD patients who met the criteria of the Diagnostic and Statistical Manual of Mental Disorders, $5^{\text {th }}$ Edition (DSM-V) were enrolled from No.102 Hospital of the Chinese People's Liberation Army from May 2014 to February 2015. Diagnoses were made independently by three attending psychiatric doctors using the Chinese version of the modified Structured Clinical Interview for DSM-V. The severity of GAD was assessed using the 14-item Hamilton Anxiety Scale (HAMA). All patients were presenting for their first visit without any prior clinical treatment. They had not taken antipsychotic, antidepressant, anxiolytic drugs for at least three months before study enrollment, had no previous history of organic disease (such as heart disease, diabetes, and $\mathrm{Pa}$ The 41 healthy controls were recruited from No.102 Hospital of the Chinese People's Liberation Army from May 2014 to Fabuary 2015., without any family history 
of major psychiatric disorders (including schizophrenia, bipolar disorder and anxiety disorders) and without any history of severe traumatic events in the past month.

Prior to study entry, all risks, benefits and potential adverse events associated with participation in the study were explained to the participants and their legal guardians, who provided written informed consent according to a protocol approved by the Ethical Committee for Medicine of No.102 Hospital of Chinese People's Liberation Army, PR, China.rkinson's disease) and no other psychiatric disorders.

Table 1: Demographic distribution of case and control group

\begin{tabular}{cccccc}
\hline Items & & GAD $(\mathbf{n = 6 9 )}$ & NC $(\mathbf{n = 4 1 )}$ & $\chi^{2} / \mathbf{t}$ value & P value \\
\hline Gender & male & $33(47.8 \%)$ & $20(48.8 \%)$ & $\chi^{2}=6.858$ & 0.487 \\
& female & $36(52.2 \%)$ & $21(51.2 \%)$ & & \\
Age & $\overline{\mathrm{X}} \pm \mathrm{s}$ & $48.81 \pm 14.99$ & $39.44 \pm 13.74$ & $\mathrm{t}=4.755$ & 0.389 \\
Urban/Rural & Urban & $39(56.5 \%)$ & - & & \\
& Rural & $30(43.5 \%)$ & - & \\
Marriage & Spinsterhood & $12(17.4 \%)$ & - & \\
& Married & $57(82.6 \%)$ & - & & \\
& & & - & & \\
\hline
\end{tabular}

\subsection{Experimental Methods}

\subsubsection{Experimental Procedures}

Hamilton Anxiety Scale9 (HAMA: The clinical symptomatology and severity of all depression patients were assessed by 3 psychiatric doctors at 0 -week, 4-week and 8-week antidepressant treatment. HAMA (Chinese version) was used to present the most items were adopted with fiverank method ("0" represents "null", "1" presents "mild", " 2 " presents "moderate", "3" presents "severe", "4" presents "extremely severe") and several items were adopted with three-rank method ("0" represents "null", "1" presents "mild moderate", "2" presents "severe"). The scale can be concluded into 7 items: anxiety/somatization, body weight, cognitive disorder, day and night change, retardation, sleep disorder and hopelessness. This scale was Chinese-localization, its reliability and validity were validated to be appreciated for Chinese population.

\subsubsection{RNA Extraction}

Whole blood $(5 \mathrm{ml})$ was collected in EDTA anticoagulant tube from each subject and processed within 1 hour. PBMCs were isolated from the blood through density gradient centrifugation and stored at $-80^{\circ} \mathrm{C}$ until use. Total RNAs were extracted from the PBMCs with Trizol(Invitrogen, Carlsbad, CA, USA) and the RNeasy kit (Qiagen, Hilden, Germany) according to the manufacturer's protocol, quantified by NanoDrop ND-2000 (Thermo Scientific, Delaware, ME, USA), DNase treated (TurboDNase, Life Technologies) and reverse transcribed (Superscript III;Invitrogen). The integration of RNA was assessed using Agilent Bioanalyzer 2100(Agilent Technologies). 


\subsubsection{Lncrna Microarray Expression Profiling}

RNA samples were used for lncRNA microarray profiling. LncRNA expression was measured by Human lncRNA 3.0 arrays (Arraystar, Santa Clara, CA, USA). The sample labeling, microarray hybridization and washing were performed based on the manufacturer's standard protocols. Afterwards, the labeled RNAs were hybridized onto the microarray. Having washed and stained the slides, the arrays were scanned by the Agilent Scanner G2505C (Agilent). The scanned images were analyzed using Feature Extraction software (version10.7.1.1, Agilent Technologies) and Genespring software (version 12.5; Agilent Technologies).

\subsubsection{Real-Time Quantitative Reverse-Transcription PCR (qRT-PCR)}

According to microarray results, the top 10 lncRNAs with the highest differentially expression was chosen for further validation with qRT-PCR. Blood samples from 69 GAD patients and 41 controls were used to validate the candidate lncRNAs. Total RNAs were isolated from the PBMCs using Trizol reagent (Invitrogen ${ }^{\circledR}$, USA) for quantitative detection of lncRNA. Complementary RNA was synthesized using the Reverse Transcription TaqMan RNA Reverse Transcription Kit (Applied Biosystems, inc., USA) according to the manufacturer's instructions. Real-time PCR was performed using Applied Biosystems 7900HT Real-Time PCR System (Applied Biosystems, Inc., USA). Data were collected using the SDS 2.3 software (Applied Biosystems, Inc.) and DataAssist v3.0 software. After normalized to $\beta$-Actin, the expression levels of lncRNAs were calculated using the $2^{-\Delta \Delta \mathrm{Ct}}$ method.

\subsubsection{Statistical Analysis}

All data were processed using DataAssist v3.0 and v17.0 of SPSS statistical software. $\Delta \mathrm{Ct}$ value was calculated on the basis of the following formula using $\beta$-Actin as an internal reference: $\Delta \mathrm{Ct}$ $=\mathrm{Ct}_{\text {lncRNA }}-\mathrm{Ct}_{\beta \text {-actin }}\left(\mathrm{Ct}\right.$, threshold cycle), $2^{-\Delta \mathrm{Ct}}$ value represented the relative expression level of lncRNAs. HAMD scale factor and total scores were compared using duplicate detecting analysis of variance at the three time points ( 0 -week, 4-week an 8-week medication treatment), and parameter detection of multiple related sample was used to compare the expression levels of lncRNAs at the three time points. What's more, Wilcoxon rank sum test was used to test the differences of eight lncRNA expression levels at the two time points randomly chosen from the three time points, moreover, Pearson correlation analysis was performed to explore the improvement of depressive symptoms and the correlations between each lncRNA. Further, multiple regression analysis was carried on to determine the accountability of lncRNAs for symptomatology improvements and ROC curve was built to explore the efficacy of lncRNA predict on depressive symptomatology. All statistical tests were two-tailed, and $\mathrm{P}<0.05$ was considered statistically significant. 


\section{Results}

\subsection{Demographic Data of the Lncrnas with Altered Expression and HAMA Items}

Psychic anxiety was positively correlated with the expression levels of PR1-PR10 ( $\mathrm{r}=0.187 \sim 0.253, P<0.01$ or $P<0.05)$, and somatic anxiety was positively correlated with the expression levels of PR7 $(r=0.171, P<0.05)$

Table 2: Correlation of lncRNAs with altered expression and HAMA items (r)

\begin{tabular}{cllllllllll}
\hline Probes & PR1 & PR2 & PR3 & PR4 & PR5 & PR6 & PR7 & PR8 & PR9 & PR10 \\
\hline $\begin{array}{c}\text { Psychic } \\
\text { anxiety }\end{array}$ & $0.187^{*}$ & $0.235^{* *}$ & $0.249^{* *}$ & $0.205^{*}$ & $0.215^{*}$ & $0.211^{*}$ & $0.253^{* *}$ & $0.215^{*}$ & $0.215^{*}$ & $0.226^{* *}$ \\
$\begin{array}{c}\text { Somatic } \\
\text { anxiety }\end{array}$ & 0.117 & 0.143 & 0.160 & 0.112 & 0.133 & 0.116 & $0.171^{*}$ & 0.128 & 0.105 & 0.141 \\
$\begin{array}{c}\text { Anxiety } \\
\text { scores }\end{array}$ & $0.195^{*}$ & $0.214^{*}$ & $0.231^{* *}$ & $0.190^{*}$ & $0.198^{*}$ & $0.194^{*}$ & $0.240^{* *}$ & $0.196^{*}$ & $0.199^{*}$ & $0.209^{*}$ \\
\hline
\end{tabular}

Note: ${ }^{*} P<0.05,{ }^{* *} P<0.01$; PR1-PR10: ENST00000505825, NONHSAT103134, NONHSAG017299, NONHSAT078768, NONHSAT029028, NONHSAT101077, NONHSAG049179, NONHSAT031726, TCONS_12_00010607, NONHSAT131696.

\subsection{Combined ROC (Receiver Operating Characteristic) Curve Analysis Of IncRNAs In GAD Patients}

To evaluate the potential of the 10 lncRNAs as biomarkers of GAD, e performed ROC analysis of as have been shown in figure 1, pre ROC analysis on PR1-10 in case group and control group showing: ROC curve analysis suggested that the AUC of PR1-10 arrived at $0.808 \quad(\mathrm{P}<0.01)$, when $\mathrm{YI}=\mathrm{YI}_{\max }$, the sensitivity as 0.663 and the specificity was 0.905 .

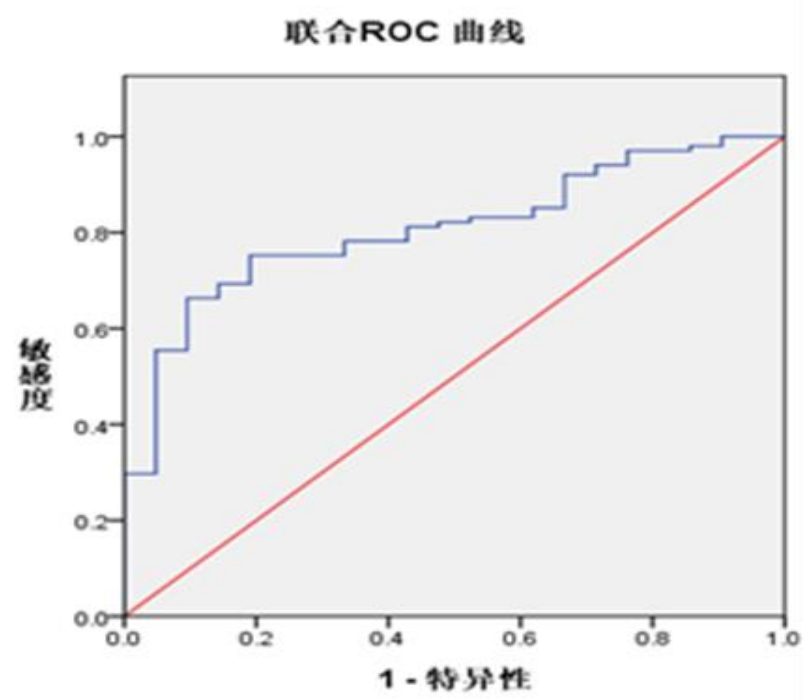

Figure 1: Combined ROC curve analysis of lncRNAs 
Table 3: Combined ROC curve results of differentially expressed lncRNAs

\begin{tabular}{|c|c|c|c|c|c|c|c|c|}
\hline \multirow[t]{2}{*}{ TargetID } & \multirow[t]{2}{*}{$\mathbf{A} \mathbf{U C}^{\mathbf{a}}$} & \multirow[t]{2}{*}{$\mathbf{S E}^{\mathbf{b}}$} & \multirow[t]{2}{*}{$P$ value } & \multicolumn{2}{|c|}{$\begin{array}{l}95 \% \text { confidence } \\
\text { interval }\end{array}$} & \multirow{2}{*}{$\mathbf{Y} \mathbf{I}_{\max }{ }^{\mathrm{e}}$} & \multicolumn{2}{|c|}{$\mathbf{Y I}=\mathbf{Y} \mathbf{I}_{\max }$} \\
\hline & & & & $\mathbf{C}_{\mathrm{L}}{ }^{\mathrm{c}}$ & $\mathrm{C}_{\mathbf{U}^{\mathrm{d}}}$ & & sensitivity & specificity \\
\hline pre ROC & 0.808 & 0.045 & 0.000 & 0.720 & 0.895 & 0.568 & 0.663 & 0.905 \\
\hline
\end{tabular}

Note: a: the combining area under the ROC curve; b: standard error; c: the lower limit of the confidence interveal; d: the higher limit of the confident interval; e: maximum of Youden index (YI, YI= sensitivity + specificity -1$)$

\subsection{Differential Scores Analysis of Anxiety Symptomatology between Lncrna High and Low Expression Group}

Recruits with scores of lncRNA expression levels higher than and lower than $27 \%$ were grouped into high and low group respectively. As shown in table 4, the psychical anxiety, somatic anxiety and anxiety scores in high expression group were significantly higher than those in low expression group $(P<0.05$ or 0.01$)$.

Table 4: Comparison of anxiety symptomatology between lncRNA high and low expression sub-

\begin{tabular}{ccccc}
\multicolumn{4}{c}{ group $(\bar{x} \pm \mathrm{S})$} & \\
\cline { 2 - 3 } $\begin{array}{c}\text { Anxiety } \\
\text { factors }\end{array}$ & $\begin{array}{c}\text { High expression } \\
\text { group }\end{array}$ & $\begin{array}{c}\text { Low expression } \\
\text { group }\end{array}$ & $\boldsymbol{t}$ & $\boldsymbol{P}$ \\
\hline $\begin{array}{c}\text { Psychical } \\
\text { anxiety }\end{array}$ & $14.73 \pm 4.69$ & $9.00 \pm 1.91$ & 46.15 & 0.000 \\
$\begin{array}{c}\text { Somatic } \\
\text { anxiety } \\
\text { Anxiety } \\
\text { scores }\end{array}$ & $14.76 \pm 3.63$ & $9.69 \pm 2.65$ & 40.38 & 0.013 \\
\hline
\end{tabular}

\section{Discussion}

Currently, the emerging trend of lncRNAs research had made it possible to tap the potential manipulating lncRNAs as a target for antipsychotic development ${ }^{[23]}$, and some RNAs had been proved to be associated with psychotic disease ${ }^{[24-25]}$. Only bioinformatics method was not enough to seek the target genes of psychotic disease, although its high specificity and sensitiveness, the consistency of prediction results were low and false positive rate was higher [26]. Through lncRNA microarray expression profiling and RT-PCR, the differential expression of IncRNAs in GAD had been already tested, and HAMA was used to assess patients' symptomatology.

lncRNA microarray expression profiling indicated that the differential expressions of lncRNAs were associated with GAD, some lncRNAs were found to be located in chromosomal region related to mental disease, such as Gomafu/MIAT/Rncr2 which can induce psychotic disease through the abnormal expression of correlation genes by inaccurate cell differentiation, stem cell 
differentiation and selective splicing. The combined curve, with area under the curve (AUC) of $0.808, \mathrm{YI}=\mathrm{YI}_{\max }$, the sensitivity and specificity of the prediction is 0.663 and 0.905 respectively, showed the important diagnosis function of these 10 lncRNAs in GAD.

Furthermore, because of the high correlation of NONHSAG049179 with symptomatology, it was singled out to analyze the relationship of the high and low score group with psychotic and somatic anxiety. Since 2015 when it was firstly reported, NONHSAG049179 has been speculated to regulate the symptomatology of anxiety. Brain, like other tissues, can combine with peripheral lymphatic system ${ }^{[31]}$. RNA regulate gene expression by combining with some enzymes and nuclear receptors at the post-transcriptional levels ${ }^{[32]}$, playing a significant role in coordinating nervous system development and neuronal function in the mature brain.

Currently, we have identified the relations of lncRNAs with the symptomatology of GAD, suggesting that these lncRNAs may have potential role in the pathogenesis of GAD. This may provide new clues for GAD research and refers for the diagnosis and personality treatment. In conclusion, the symptomatic analysis suggested that these lncRNAs may be involved in the pathogenesis of GAD.

\section{Role of Funding Source}

No funding had been received

\section{Contributors}

Ming-jun He developed the clinical trial design, collected and organized the subject data, and wrote the first draft of the manuscript. Wei Niu collected and organized the data, and participated in the critical discussion of the study design. Xiao-li Zhu, Ling-ming Kong, Gao-feng Yao collected and organized the information data. Li-yi Zhang came up with the general study design.

\section{Conflict of Interest}

All authors declare that they have no conflicts of interest.

\section{Acknowledgements}

We sincerely thank the patients, their families, and the healthy volunteers for their participation, as well as the medical staff involved in collecting the specimens.

\section{References}

[1] Liyi Zhang, Hongsheng Chen. Clinical psychology(DSM-V)[M], Beijing: People's Military Medical Publisher,2015

[2] American Psychiatric Association. Diagnostic and statistical manual of mental disorders (5 $5^{\text {th }}$ ed.) [M]. American Psychiatric Association, 2013: 145-165.

[3] Bandelow B, Lichte $\mathrm{T}$, Rudolf $\mathrm{S}$, et al. The diagnosis of and treatment recommendations for anxiety disorders [J]. DeutschesArzteblatt international, 2014; 111:473-80. 
[4] Sheng-dong Chen, Xin-yang Sun, Wei Niu, et al. Correlation between the level of microRNA expression in peripheral blood mononuclear cells and symptomatology in patients with generalized anxiety disorder [J]. Science Direct, 2016: 216-224.

[5] Kunugi H,Hori H,Numakawa T,et al. The hypothalamic-pituitary-adrenal axis and depressive disorder: recent progress [J]. Nihon ShinkeiSeishinYakurigaku Zasshi.2012, Aug 32(4):203-209.

[6] Huiming Fan, Wei Niu, Mingjun He, et al. Bioinformatics analysis of microRNA in peripheral blood of GAD patients [J], China Journal of Medical Genetics, 2015, 32 （5） : 641-646.

[7] Okazaki Y, Furuno M, Kasukawa T, et al. Analysis of the mouse transcriptome based on functional annotat ion of 60, 770 full- length cDNAs[J]. Nature, 2002, 420: 563-573.

[8] G Botti, L Marra, MG Malzone, et al. LncRNA HOTAIR as prognostic circulating marker and potential therapeutic target in patients with tumor diseases[J]. Current Drug Targets, 2017, 18(1):27.

[9] S Yotsukura, D Duverle, T Hancock, et al. Computational recognition for long non-coding RNA (lncRNA): Software and databases [J]. Briefings in Bioinformatics, 2017, 18(1):9.

[10] U Perron, P Provero, I Molineris. In silico prediction of lncRNA function using tissue specific and evolutionary conserved expression [J]. Bmc Bioinformatics, 2017, 18(5):144.

[11] YA Huang, X Chen, ZH You, et al. ILNCSIM: improved lncRNA functional similarity calculation model [J]. Oncotarget, 2016, 7(18):25902-25914.

[12] A Bhan, SS Mandal. LncRNA HOTAIR: A master regulator of chromatin dynamics and cancer [J]. BiochimicaEtBiophysicaActa, 2015, 1856(1):151-64.

[13] MD Ballantyne, RAMcdonald, AH Baker. LncRNA/microRNA interactions in the vasculature [J]. Clinical Pharmacology \& Therapeutics, 2016, 99(5):494-501.

[14] Clark B S. Long non-coding RNA-dependent transcriptional regulation in neuronal development and disease [J]. Non-Coding RNA, 2014, 5:164-164.

[15] M Sun, FQ Nie, ZX Wang, et al. Involvement of lncRNAdysregulation in gastric cancer [J]. Histology \& Histopathology, 2016, 31(1):33.

[16] Barry G, Briggs J A, Vanichkina D P, et al. The long non-coding RNA Gomafu is acutely regulated in response to neuronal activation and involved in schizophrenia-associated alternative splicing [J]. Molecular Psychiatry, 2014, 19(4):486-494.

[17] Rao S Q, Hu H L, Ning Y, et al. Genetic variants in long non-coding RNA MIAT contribute to risk of paranoid schizophrenia in a Chinese Han population.[J]. Schizophrenia Research, 2015.

[18] Yang X, Gao L, Guo X, et al. A Network Based Method for Analysis of lncRNA-Disease Associations and Prediction of lncRNAs Implicated in Diseases [J]. Plos One, 2014, 9(1):e87797.

[19] Lau E. Non-coding RNA: Zooming in on lncRNA functions [J]. Nature Reviews Genetics, 2014, 15(9):574-575.

[20] Hong-tao Song, Xin-yang Sun, Liang Zhang, et al. A preliminary analysis of association between the down-regulation of microRNA-181b expression and symptomatology improvement in schizophrenia patients before and after antipsychotic treatment [J]. Journal of Psychiatric Research, 2014, 54(7):134-140.

[21] Bin Xu, Pei-Ken Hsu, Kimberly L. Stark,et al. DE repression of a Neuronal Inhibitor due to miRNADys regulation in a Schizophrenia-Related Micro deletion[J]. Cell, 2013, 152(1-2): 262275.

[22] Yuhua Tang, Mingyuan Zhang. Hamilton Anxiety Scale (HAMA)[J]. Shanghai Archives of Psychiatry, 1984, (2).

[23] Cui X, Niu W, Kong L, He M, Jiang K, Chen S, Zhong A, Li W, Lu J, Zhang L. hsa_circRNA_103636: potential novel diagnostic and therapeutic biomarker in Major depressive disorder [J]. Biomark Med, 2016, 10(9):943-952.

[24] Fan HM, Sun XY, Guo W, Zhong AF, Niu W, Zhao L, et al. Differential expression of microRNA in peripheral blood mononuclear cells as specific biomarker for major depressive disorder patients [J]. Journal of psychiatric research, 2014; 59:45-52. 
[25] Fan H-m, Sun X-y, Guo W, Zhong A-f, Niu W, Zhao L, et al. Differential expression of microRNA in peripheral blood mononuclear cells as specific biomarker for major depressive disorder patients [J]. Journal of psychiatric research, 2014.

[26] $\mathrm{Xu} \mathrm{X}$. Same computational analysis, different miRNA target predictions [J]. Nature methods, 2007; 4:191.

[27] Fan HM, Niu W, He MJ, Zhong AF, Zhang QL, Yan Y, et al. Bioinformatics analysis of differently expressed microRNAs in anxiety disorder [J]. Med Genet 2015; 32:641-6.

[28] Ishizuka A, Hasegawa Y, Ishida K, Yanaka K, Nakagawa S. Formation of nuclear bodies by the lncRNAGomafu-associating proteins Celf3 and SF1[J]. Genes Cells, 2014, 19(9): 704-721.

[29] JD Mills, J Chen, WS Kim, et al. long intervening non-coding RNA 00320 is human brainspecific and highly expressed in the cortical white matter [J]. Neurogenetics, 2015, 16(3):1-13.

[30] JihuaNie, ChaojunPeng, Weiwei Pei, et al. A novel role of long non-coding RNAs in response to X-ray irradiation [J]. Toxicology in Vitro, 2015;DIO:10.1016/j.tiv.2015.09.007.

[31] Louveau A, Smirnov I, Keyes TJ, et al. Structural and functional features of central nervous system lymphatic vessels. [J]. Nature, 2015.

[32] Hansen T B, Jensen T I, Clausen B H, et al. Natural RNA circles function as efficient microRNA sponges [J]. Nature, 2013, 495(7441): 384-388.

* Joint correspondence authors

The author who is to receive correspondence and reprint requests:

Liyi Zhang, E-mail:zly102@126.com. Prevention and Treatment Center for Psychological Diseases, No.102

Hospital of Chinese People's Liberation Army, North Peace Road 55, People's Republic of China. Tel: +86

519 83064556; fax: +8651983064560.

E-mail addresses: chado808@126.com (S-d.Chen) ; 339231442@qq.com (M-j.He), 156252143@qq.com (XL Zhu); niuwei102@126.com (W. Niu), lingmk123@163.com (L-m.Kong), 18915027113@126.com (G-f Yao),zly102@126.com (L-y.Zhang) 